\title{
Clusters as surrogate for explicit short-range correlations in relativistic mean-field models
}

\author{
Stefan Typel ${ }^{\mathrm{a}}$ \\ Technische Universität Darmstadt, Fachbereich Physik, Institut für Kernphysik, \\ Schlossgartenstraße 9, 64289 Darmstadt, Germany \\ GSI Helmholtzzentrum für Schwerionenforschung GmbH, Theorie, Planckstraße 1, \\ 64291 Darmstadt, Germany
}

Received 9 April 2020 / Accepted 27 October 2020

Published online 21 December 2020

\begin{abstract}
The formation of clusters at sub-saturation densities in nuclear matter can be seen as a result of many-body correlations. Various theoretical models have been developed to take this effect into account, mostly on a phenomenological level using energy density functionals. These models are constructed in such a way that clusters appear solely in dilute matter and dissolve when the density approaches the nuclear saturation density. At higher densities only nucleons survive as quasi-particles and no explicit correlations between the constituents of nuclear matter are considered. The possible description of correlations with cluster degrees of freedom at supra-saturation densities is explored using the example of a quasi-deuteron in a generalized relativistic density functional. The required change in the density dependence of the cluster mass shift, responsible for describing the cluster dissolution in the present model, is derived for nuclear matter at zero temperature.
\end{abstract}

\section{Introduction}

Short-range correlations between nucleons are an essential feature in strongly interacting systems [1,2]. They originate from the repulsive hard-core of the nuclear interaction and the short-range attraction in specific channels. These correlations appear not only in nuclei but they can also affect the thermodynamic properties of nuclear matter in a large range of baryon density, temperature and isospin asymmetry in the phase diagram. Even the chemical composition can be modified due to the appearance of many-body bound states, i.e. nuclei. This cluster formation is particularly crucial for applications in astrophysics where the equation of state is an important ingredient in the determination of compact-star properties or in simulations of core-collapse supernovae and neutron-star mergers [3].

Many methods have been developed in nuclear physics in order to take shortrange correlations into account in theoretical calculations using realistic nucleonnucleon interactions, see, e.g., [3-5] and references therein. Alternatively, effective field-theoretical methods are employed that are guided by symmetry principles from

\footnotetext{
a e-mail: stypel@ikp.tu-darmstadt.de
} 
QCD and that explore the concept of scale separation using systematic expansions in powers of small parameters $[2,6]$.

Another line of approaches are energy density functionals (EDFs) which are usually derived in self-consistent mean-field approximation with an effective in-medium interaction [7]. They are more phenomenological models without a direct connection to the nucleon-nucleon interaction in free space. Instead, their parameters are determined from directly fitting properties of finite nuclei and sometimes characteristic parameters of nuclear matter near saturation, e.g., the binding energy per nucleon, the saturation density or the incompressibility. There are various versions of this approach, e.g., non-relativistic models using Skyrme or Gogny type interactions $[8,9]$ or relativistic models based on the exchange of mesons [10]. In general, only baryons are the relevant degrees of freedom in these EDFs. They become quasi-particles in the medium with modified properties as compared to the vacuum. However, there are no explicit correlations between these quasi-particles.

About a decade ago, interacting clusters were introduced as explicit degrees of freedom in relativistic density functionals, concentrating on the light clusters ${ }^{2} \mathrm{H},{ }^{3} \mathrm{H}$, ${ }^{3} \mathrm{He}$ and ${ }^{4} \mathrm{He}$ at first [11]. This generalization of standard EDFs is quite natural since the description of very dilute matter as an ideal mixture of nucleons and all nuclei from the table of isotopes in thermodynamic equilibrium, usually called nuclear statistical equilibrium (NSE), is widely used in astrophysics. This approximation, however, is valid only as long as the interaction between the constituents can be neglected. There is even an exact low-density limit at finite temperatures, the virial equation of state [12-15]. It can take interaction effects into account and depends only on experimental observables like binding energies and scattering phase shifts. A generalization based on a quantum statistical approach has been developed [16-22].

But in simple models with cluster degrees of freedom a new problem arises. With increasing density, the system approaches nuclear saturation density. It is expected that all clusters dissolve in the medium so that only nucleons remain at higher densities. This is not realized in a standard NSE approach. Instead a conversion of the matter to a mixture of heavier and more strongly bound nuclei is found. There are two main methods to describe the dissolution of clusters with increasing density. The traditional excluded-volume approach uses a simple geometric picture [23-26]. A finite size is assigned to every cluster so that they cannot exist above a certain density since the maximum packing fraction is reached. In Figure 1 a simple example for the deuteron mass fraction $X_{d}=2 n_{d} / n_{b}$ in symmetric neutron-proton-deuteron matter is depicted. Here, $n_{d}$ and $n_{b}=n_{n}+n_{p}+2 n_{d}$ are the deuteron number density and the total baryon number density, respectively. NSE calculations (dashed lines) for different temperatures are compared to calculations assuming a finite volume of the deuteron (full lines). The effect of the deuteron suppression with increasing density is obvious, in particular the rapid decrease of $X_{d}$ when a certain limiting density is approached from below, independent of the temperature.

A more recent approach to describe the dissolution of clusters uses a microscopically motivated method. The basic idea is that the properties of the clusters can change in the medium, in particular the mass or binding energy. This effect will depend not only on the density but also on temperature and even the momentum of the cluster. The size of this mass shift can be calculated employing a many-body Schrödinger equation with appropriate potentials taking the medium into account $[16,17,22]$. One essential feature of this approach is the Pauli exclusion principle. The formation of clusters is suppressed when a considerable fraction of the available single-particle states in momentum space is already occupied and is no longer available for the cluster. This Pauli blocking is less strong when the surface of the Fermi sphere becomes more dilute at higher temperatures or the center-of-mass momentum of the cluster is much larger than the typical radius of this sphere. The mass shifts from microscopic calculations can be parametrized using simple functional forms with 


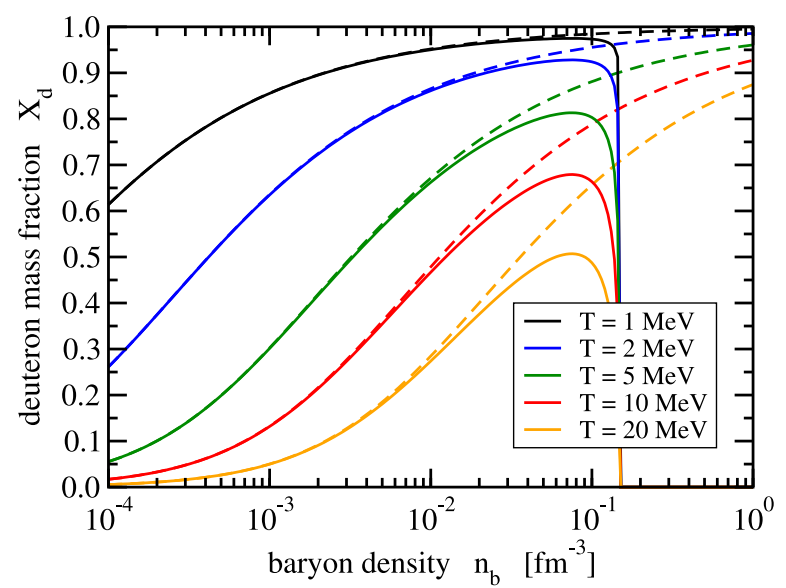

Fig. 1. Deuteron mass fraction $X_{d}$ in isospin symmetric neutron-proton-deuteron matter in a NSE model without (dashed lines) and with (full lines) excluded-volume mechanism as a function of the total baryon number density $n_{b}$ for different temperatures from $1 \mathrm{MeV}$ (top) to $20 \mathrm{MeV}$ (bottom).

appropriate extrapolations to high densities $[11,22,27,28]$. Then they are used in the EDF for the calculation of the system properties, e.g., the equation of state of nuclear matter. Details for light clusters will be given in Section 2. When the effective inmedium binding energy of a cluster vanishes, at the so-called Mott density, the cluster will dissolve since no bound state exists any more.

In the existing EDFs with the excluded-volume mechanism or with mediumdependent mass shifts, all clusters disappear above nuclear saturation density by construction and a simple system of neutron and proton quasi-particles remains. But it is well known that short-range correlations between nucleons also exist at supra-saturation densities. One experimental evidence is found from nucleon knockout reactions on nuclei using inelastic electron scattering, see [1] and references therein. A sizable fraction of correlated nucleon-nucleon pairs is observed. This causes an extended tail in the single-nucleon momentum distribution. It decreases approximately proportional to the inverse fourth power $\left(\propto k^{-4}\right)$ of the momentum $k$. Interestingly, the absolute height of these tails is practically identical for neutrons and protons even in a neutron-proton asymmetric system. Thus a stronger enhancement of the tail of the minority species as compared to the majority species is observed [29].

Considering the discussion above, the question arises whether clusters can be used as a surrogate for an explicit treatment of short-range correlations in EDFs. Since the many-body wave function of a cluster contains correlated nucleons, this would leave an imprint on the single-nucleon momentum distribution of dense nuclear matter even at zero temperature. In order to find these quasi-clusters above the saturation density, a substantial modification of the cluster mass shift has to be expected. In this work, the necessary modifications of a relativistic EDF with clusters above saturation will be investigated concentrating on deuteron-like clusters. The relevant condition on the mass shift will be formulated and compared to the traditional treatment.

This work is structured as follows. In Section 2 the basics of the generalized relativistic density functional (GRDF) are given with particular attention to the mass shifts of light clusters and the extension to zero temperature. The dependence of the mass shifts on the density above saturation is discussed using as example the deuteron. Conditions on the deuteron mass shift in symmetric nuclear matter 
are discussed in Section 3 if the deuteron is used as an effective means to include short-range nucleon-nucleon correlations. Conclusions and an outlook are given in Section 3 .

\section{Relativistic density functional with clusters}

The GRDF is an extension of a conventional relativistic mean-field model with nucleons by adding light clusters $\left(d={ }^{2} \mathrm{H}, t={ }^{3} \mathrm{H}, h={ }^{3} \mathrm{He}, \alpha={ }^{2} \mathrm{He}\right)$ as explicit degrees of freedom [11]. The effective in-medium interaction between nucleons, free or bound in clusters, is described by the exchange of mesons with density dependent couplings. The mass-shift approach is used to obtain the dissolution of clusters with increasing density. Here we follow the notation in [30] where more details can be found.

\subsection{Fundamental quantities of the GRDF}

The model for nuclear matter is introduced in a thermodynamic formulation by defining a total grand canonical potential density $\omega$ that depends on the temperature $T$ and the chemical potentials $\mu_{i}$ of all constituents, i.e., nucleons, $d, t, h$, and $\alpha$. Since the case of zero temperature will be considered in this work, the standard form of $\omega$ as given in [30] for finite temperatures has to be generalized by adding a term that considers the contribution of bosonic particles in condensates. Thus it is given as

$$
\omega\left(T,\left\{\mu_{i}\right\}\right)=\sum_{i}\left(\omega_{i}+\frac{1-\sigma_{i}}{2} \omega_{i}^{(\mathrm{cond})}\right)+\omega_{\text {meson }}-\omega_{\text {meson }}^{(r)}-\omega_{\text {mass }}^{(r)}
$$

with the standard non-mesonic quasi-particle contribution

$$
\omega_{i}\left(T, m_{i}^{*}, \mu_{i}^{*}\right)=-T \frac{g_{i}}{\sigma_{i}} \int \frac{d^{3} k}{(2 \pi)^{3}} \ln \left\{1+\sigma_{i} \exp \left[-\frac{E_{i}\left(k, m_{i}^{*}\right)-\mu_{i}^{*}}{T}\right]\right\}
$$

a condensate contribution, only relevant for bosons,

$$
\omega_{i}^{(\mathrm{cond})}=g_{i} \xi_{i}\left(m_{i}^{*}-\mu_{i}^{*}\right)
$$

and three additional terms: the contribution of mesons, a rearrangement contribution due to the density dependence of the nucleon-meson couplings and a term arising from the density dependence of the cluster mass shifts. Explicit expressions for $\omega_{\text {meson }}$, $\omega_{\text {meson }}^{(r)}$, and $\omega_{\text {mass }}^{(r)}$ can be found in [30]. The sign $\sigma_{i}$ distinguishes between fermions $\left(\sigma_{i}=+1\right)$ and bosons $\left(\sigma_{i}=-1\right)$. In equation (2) the degeneracy factor $g_{i}$ and the energy $E_{i}=\sqrt{k^{2}+\left(m_{i}^{*}\right)^{2}}$ of a quasi-particle $i$ appear. The factor $\xi_{i}$ in equation (3) quantifies the size of the condensate contribution, see below. The effective mass $m_{i}^{*}=m_{i}-S_{i}$ and effective chemical potential $\mu_{i}^{*}=\mu_{i}-V_{i}$ contain scalar and vector potentials that are given by

$$
S_{i}=C_{\sigma} g_{i \sigma} n_{\sigma}+C_{\delta} g_{i \delta} n_{\delta}-\Delta m_{i}=S_{i}^{(\text {meson })}-\Delta m_{i}
$$

and

$$
V_{i}=C_{\omega} g_{i \omega} n_{\omega}+C_{\rho} g_{i \rho} n_{\rho}+B_{i} V^{(r)}+W_{i}^{(r)}=V_{i}^{(\text {meson })}+W_{i}^{(r)}
$$


respectively, if a vector density dependence of the nucleon meson couplings $\Gamma_{m}$ in $C_{m}=\Gamma_{m}^{2} / m_{m}^{2}$ is assumed. Here, four types of mesons $m$ are considered: $\sigma, \omega, \rho$, and $\delta$ with the corresponding source densities $n_{m}$. The DD2 parametrization [11] of the couplings is used. The factors $g_{i m}$ define the strength of the coupling between a quasiparticle $i$ and a meson $m$ and $B_{i}$ is the baryon number. The quantities $\Delta m_{i}, V^{(r)}$, and $W_{i}^{(r)}$ are the mass shift, the rearrangement term due to the density dependence of $\Gamma_{m}$, and the mass-shift rearrangement contribution. All relevant equations and quantities can be derived from the potential density (1).

The quasi-particle number densities (= vector densities) are found as

$$
n_{i}=-\left.\frac{\partial \omega}{\partial \mu_{i}}\right|_{T,\left\{\mu_{j}\right\}_{j \neq i}}=n_{i}^{(v)}=g_{i} \int \frac{d^{3} k}{(2 \pi)^{3}} d_{i}\left(T, k, m_{i}^{*}, \mu_{i}^{*}\right)+\frac{1-\sigma_{i}}{2} g_{i} \xi_{i}
$$

from the proper derivatives of (1) with respect to the chemical potentials $\mu_{i}$ with the distribution function

$$
d_{i}=\left\{\exp \left[\frac{E_{i}\left(k, m_{i}^{*}\right)-\mu_{i}^{*}}{T}\right]+\sigma_{i}\right\}^{-1}
$$

The rearrangement terms in the vector potential (5) are essential to obtain this standard form for the densities. From a differentiation of (1) with respect to the particle vacuum masses $m_{i}$, the scalar densities

$$
n_{i}^{(s)}=\left.\frac{\partial \omega}{\partial m_{i}}\right|_{T,\left\{\mu_{j}\right\}}=g_{i} \int \frac{d^{3} k}{(2 \pi)^{3}} \frac{m_{i}^{*}}{E_{i}} d_{i}\left(T, k, m_{i}^{*}, \mu_{i}^{*}\right)+\frac{1-\sigma_{i}}{2} g_{i} \xi_{i}
$$

are obtained. The condensate density $n_{i}^{(c)}=g_{i} \xi_{i}$ gives identical contributions to the vector and scalar densities unlike the standard thermal parts that vanish for $T \rightarrow 0$.

\subsection{Mass shifts of light clusters}

The suppression of cluster formation in the GRDF is achieved by introducing the mass shifts $\Delta m_{i}$ in the scalar potentials (4) for all clusters composed of nucleons. In general there are several contributions to $\Delta m_{i}$, e.g., from the Pauli blocking of states in nuclear matter or a change of the cluster binding energy in compact star matter where the Coulomb interaction is screened by the electronic background. In the case of nuclear matter, the electromagnetic interaction is not taken into account and there are no electrons included to compensate the charge of protons to achieve charge neutrality. Hence, the total mass shifts is just the strong mass shifts $\Delta m_{i}^{\text {(strong) }}$ from Pauli blocking.

The strong mass shifts can be calculated explicitly for various conditions of temperature, density and isospin asymmetry of the medium by solving the inmedium many-body Schrödinger equation with proper potentials containing factors that take the Pauli blocking into account. These results can be approximated by more-or-less simple functions over a large range of thermodynamic variables. Several parametrizations have been developed $[11,22,27,28]$. Here the form

$$
\Delta m_{i}^{(\text {strong })}\left(T,\left\{n_{i}^{(v)}\right\}\right)=f_{i}\left(n_{i}^{(\text {eff })}, n_{i}^{(\text {diss })}\right) B_{i}^{(0)}
$$


with a shift function $f_{i}$ and the cluster binding energy $B_{i}^{(0)}$ in vacuum is used. The shift function depends on all quasi-particle vector densities $n_{i}^{(v)}$ via the effective density

$$
n_{i}^{(\mathrm{eff})}=\frac{2}{A_{i}}\left[Z_{i} Y_{q}+N_{i}\left(1-Y_{q}\right)\right] n_{b}
$$

and on the temperature $T$ via the dissociation density

$$
n_{i}^{(\mathrm{diss})}=\frac{B_{i}^{(0)}}{\delta B_{i}(T)} .
$$

A dependence of the mass shift on the momentum of the cluster with respect to the medium is neglected in this parametrization for simplicity. Explicit expressions for $\delta B_{i}(T)$ are given in [11]. They regulate the temperature dependence of the mass shifts. In the limit $T \rightarrow 0$, the expressions of equation (26) and (27) of reference [11] reduce to $\delta B_{i}(0)=a_{i, 1} /\left(2 a_{i, 2}^{3 / 2} a_{i, 3}^{2}\right)=3634.16 \mathrm{MeV} \mathrm{fm}^{3}$ for $i=d$ and $\delta B_{i}(0)=a_{i, 1} / a_{i, 2}^{3 / 2}=$ $3389.70,3901.00,4715.99 \mathrm{MeV} \mathrm{fm}^{3}$ for the light clusters $i=t, h, \alpha$ with parameters $a_{i, 1}, a_{i, 2}, a_{i, 3}$ given in table I of reference [11]. The effective density (10) introduces a dependence of the mass shifts on the isospin asymmetry of the system. In equation (10), the charge number $Z_{i}$, the neutron number $N_{i}$, and the mass number $A_{i}=$ $Z_{i}+N_{i}$ of the cluster $i$ enter as well as the baryon number density $n_{b}=\sum_{i} B_{i} n_{i}^{(v)}$ with baryon numbers $B_{i}=A_{i}$ and the hadronic charge fraction $Y_{q}=\sum_{i} Z_{i} n_{i} / n_{b}$ of the system. In symmetric nuclear matter with $Y_{q}=1 / 2$ one has $n_{i}^{(\text {eff })}=n_{b}$. In this work the particular functional form

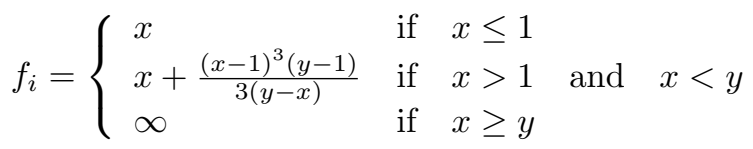

with the two parameters

$$
x=\frac{n_{i}^{(\mathrm{eff})}}{n_{i}^{(\mathrm{diss})}} \quad y=\frac{n_{\mathrm{sat}}}{n_{i}^{(\mathrm{diss})}}
$$

is used for the shift function as introduced in [30]. With this choice a linear increase of the mass shift with $n_{b}$ at low baryon densities is described consistent with the results obtained in $[11,22,27,28]$. As soon as the effective density becomes larger than the dissociation or Mott density, i.e. $x>y$, a stronger density dependence is assumed. This extrapolation is only heuristic in order to prevent the clusters to reappear at higher baryon densities.

The density dependence of the mass shifts of the four light clusters in the GRDF model is depicted in Figure 2 for symmetric nuclear matter at zero temperature. The change of the slope clearly shows the transition from the linear to the cubic dependence. This happens for the deuteron at a much lower baryon density as compared to $t, h$, and the $\alpha$ cluster. This transition point is defined by the vanishing of the effective binding energy $B_{i}^{(\text {eff })}=B_{i}^{(0)}-\Delta m_{i}$ as shown in Figure 3. Obviously, $B_{i}^{(\text {eff })}$ becomes identical to $B_{i}^{(0)}$ in the limit $n_{b} \rightarrow 0$. The strong increase of the mass shifts for $n_{b}$ approaching the nuclear saturation density $n_{\text {sat }}=0.149065 \mathrm{fm}^{-3}$ of the GRDF model with parametrization DD2 leads to a strong suppression of their densities so 


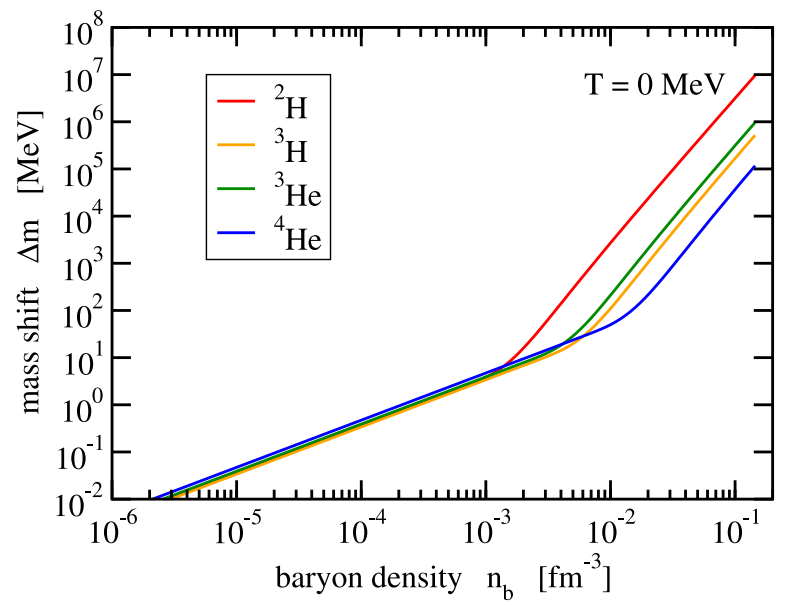

Fig. 2. Mass shifts $\Delta m$ of light clusters at zero temperature in isospin symmetric nuclear matter as function of the total baryon number density in the GRDF model with the parametrizations (9).

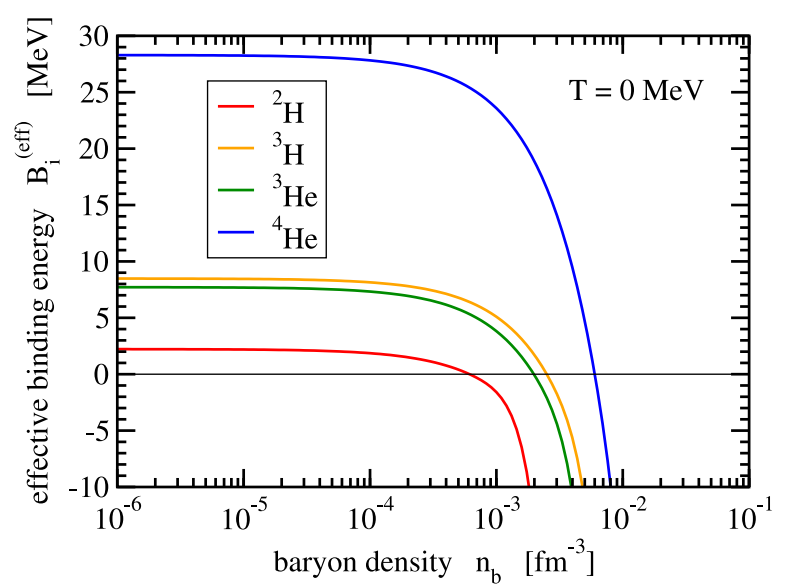

Fig. 3. Effective binding energies $B_{i}^{(\text {eff })}$ of light clusters at zero temperature in isospin symmetric nuclear matter as function of the total baryon number density in the GRDF model.

that they are removed from the system and only matter composed of neutrons and protons remains above saturation.

\section{Quasi-deuterons above saturation}

With the basic quantities and parametrizations of the GRDF model with light clusters defined in the previous section, a possible modification of the cluster mass shifts can be investigated so that they can be used as an effective means to model nucleonnucleon correlations. Since two-body short-range correlations in the $\mathrm{np}^{3} S_{1}$ channel are much more important than other many-body correlations, the deuteron is chosen as example. Of course, the deuteron inside the medium will have a different wave 
function as compared to the deuteron in vacuum but here the main interest is in the size of the deuteron mass shift.

The chemical composition of the system is defined by the total baryon density

$$
n_{b}=n_{n}+n_{p}+A_{d} n_{d}
$$

and the hadronic charge fraction

$$
Y_{q}=\frac{n_{p}+Z_{d} n_{d}}{n_{b}}
$$

under the condition of chemical equilibrium, i.e., the relation

$$
\mu_{d}=N_{d} \mu_{n}+Z_{d} \mu_{p}
$$

of the chemical potentials has to hold. In the following the case of zero temperature is assumed. Then the neutron and proton densities are given by $n_{n}=g_{n} k_{n}^{3} /\left(6 \pi^{2}\right)$ and $n_{p}=g_{p} k_{p}^{3} /\left(6 \pi^{2}\right)$ with Fermi momenta $k_{n}, k_{p}$ and degeneracy factors $g_{n}=g_{p}=2$. The deuteron density $n_{d}=d_{d} \xi_{d}$ with $g_{d}=3$ is given by the contribution from the condensate term only. The factor $\xi_{d}$ is not apriori given but cannot have any value because the deuteron density has to lie between $n_{d}^{(\mathrm{min})}=0$ and

$$
n_{d}^{(\max )}=\min \left[\frac{Y_{q} n_{b}}{Z_{d}}, \frac{\left(1-Y_{q}\right) n_{b}}{N_{d}}\right]
$$

since at $T=0$ all neutron (protons) will be bound inside the deuteron for neutron-rich (proton-rich) matter. The relation of the chemical potentials (16) can be expressed as

$$
m_{d}^{*}+V_{d}=N_{d}\left[\sqrt{k_{n}^{2}+\left(m_{n}^{*}\right)^{2}}+V_{n}\right]+Z_{d}\left[\sqrt{k_{p}^{2}+\left(m_{p}^{*}\right)^{2}}+V_{p}\right]
$$

where the mass shift of the deuteron enters via the effective mass of the deuteron $m_{d}^{*}=m_{d}-S_{i}$ and the mass rearrangement terms

$$
W_{i}^{(r)}=\sum_{j=n, p, d} n_{j}^{(s)} \frac{\partial \Delta m_{j}}{\partial n_{i}^{(v)}}=n_{d}^{(s)} \frac{\partial \Delta m_{d}}{\partial n_{i}^{(v)}}
$$

for $i=n, p, d$ in the vector potentials (5). Thus equation (18) is a linear first-order partial differential equation for $\Delta m_{d}$ in general.

Equation (18) simplifies considerably for specific choices of the coupling factors $g_{i m}$ in equations (4) and (5) and of the density dependence of the mass shift (9). It is natural to assume that the nucleons inside the deuteron couple to the Lorentz vector mesons with the same strength as the free nucleons. Hence

$$
g_{d \omega}=N_{d}+Z_{d}=2 \quad g_{d \rho}=N_{d}-Z_{d}=0
$$

for the deuteron and

$$
g_{n \omega}=g_{p \omega}=1 \quad g_{n \rho}=-g_{p \rho}=1
$$

for the nucleons. Then equation (18) reduces to

$$
m_{d}+\Delta m_{d}
$$




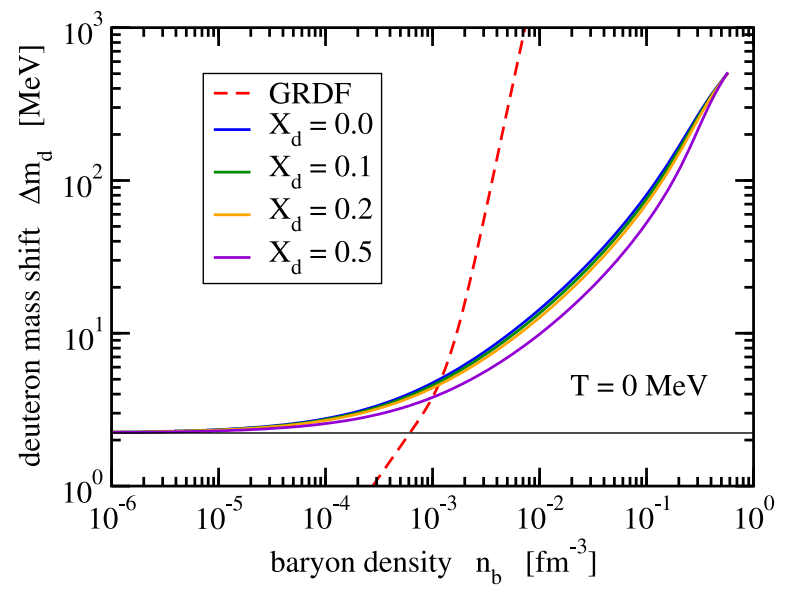

Fig. 4. Deuteron mass shift $\Delta m_{d}$ at zero temperature in isospin symmetric matter in the original GRDF model (red dashed line) in comparison with mass shifts from equation (25) to find a given deuteron mass fraction $X_{d}$ (colored full lines). The horizontal black line indicates the experimental deuteron binding energy in vacuum. See text for details.

$$
\begin{aligned}
= & S_{d}^{(\text {meson })}+N_{d} \sqrt{k_{n}^{2}+\left(m_{n}-S_{n}^{(\text {meson })}\right)^{2}}+Z_{d} \sqrt{k_{p}^{2}+\left(m_{p}-S_{p}^{(\text {meson })}\right)^{2}} \\
& +N_{d} W_{n}^{(r)}+Z_{d} W_{p}^{(r)}-W_{d}^{(r)}
\end{aligned}
$$

without the mesonic contributions to the vector potentials. The density dependence of the deuteron mass shift is a function of only the effective density

$$
n_{d}^{(\mathrm{eff})}=\frac{2}{A_{d}}\left[Z_{d}\left(n_{p}+Z_{d} n_{d}\right)+N_{d}\left(n_{n}+N_{d} n_{d}\right)\right]
$$

and thus the term

$$
\begin{aligned}
N_{d} W_{n}^{(r)}+Z_{d} W_{p}^{(r)}-W_{d}^{(r)} & =n_{d}^{(s)} \frac{\partial \Delta m_{d}}{\partial n_{d}^{(\mathrm{eff})}}\left(N_{d} \frac{\partial n_{d}^{(\mathrm{eff})}}{\partial n_{n}^{(v)}}+Z_{d} \frac{\partial n_{d}^{(\mathrm{eff})}}{\partial n_{p}^{(v)}}-\frac{\partial n_{d}^{(\mathrm{eff})}}{\partial n_{d}^{(v)}}\right) \\
& =n_{d}^{(s)} \frac{\partial \Delta m_{d}}{\partial n_{d}^{(\mathrm{eff})}} \frac{2}{A_{d}}\left[N_{d}^{2}+Z_{d}^{2}-\left(Z_{d}^{2}+N_{d}^{2}\right)\right]=0
\end{aligned}
$$

with the derivatives vanishes. Then the deuteron mass shift is only a simple function

$$
\begin{aligned}
\Delta m_{d}= & S_{d}^{(\text {meson })}-m_{d} \\
& +N_{d} \sqrt{k_{n}^{2}+\left(m_{n}-S_{n}^{(\text {meson })}\right)^{2}}+Z_{d} \sqrt{k_{p}^{2}+\left(m_{p}-S_{p}^{(\text {meson })}\right)^{2}} .
\end{aligned}
$$

For a given baryon density $n_{b}$, hadronic charge fraction $Y_{q}$, and deuteron mass fraction $X_{d}=2 n_{d} / n_{b}$, the number densities of $n, p$, and $d$ are fixed and the deuteron mass shift can be calculated explicitly in this way assuming $T=0$ and that the deuteron contribution arises exclusively from the condensate.

The case of symmetric nuclear matter at zero temperature is studied in Figure 4. The deuteron mass shift in the parametrization of the original GRDF model [11] is depicted as the red dashed line, corresponding to the full red line in Figure 2. It starts with a vanishing mass shift at zero baryon density and then increases with the 
density of the medium. It rises particularly fast when the deuteron binding energy in vacuum, the horizontal black line, is crossed at the dissociation density of $n_{d}^{\text {(diss) }}=$ $6.1 \times 10^{-4} \mathrm{fm}^{-3}$. The density dependence of the deuteron mass shift is rather different when it is calculated from equation (25) assuming the condition of condensation. In symmetric nuclear matter at zero temperature the deuteron mass fraction can have any value between zero and $X_{d}^{(\max )}=2 n_{d}^{(\max )} / n_{b}=1$. The result for $\Delta m_{d}$ depends on the assumed deuteron fraction $X_{d}$ in nuclear matter because the Fermi momenta of the nucleons and the scalar potentials of the particles are different. The full lines in Figure 4 show the dependence for four values $X_{d}=0.0,0.1,0.2$, and 0.5 with the largest $X_{d}$ corresponding to the lowest mass shift. In the low-density limit, the quasideuteron mass shift (25) approaches the deuteron binding energy in vacuum because $\mu_{n}=m_{n}, \mu_{p}=m_{p}$, and $\mu_{d}=m_{d}+\Delta m_{d}=m_{n}+m_{p}-B_{d}^{(0)}+\Delta m_{d}=\mu_{n}+\mu_{p}$. At high baryon densities above approx. $10^{-4} \mathrm{fm}^{-3}$ these mass shifts for the condensate condition are lower than that of the original GRDF parametrization. A comparison of the full blue line in Figure 4 for $X_{d}=0.0$, i.e., the onset condition for condensation, and the red dashed line of the original GRDF mass shift is of particular interest. At high densities $\mu_{d}=m_{d}+\Delta m_{d}-S_{d}^{(\text {meson) }} \gg \mu_{n}+\mu_{p}$ and there will be no deuterons at all in the system as expected. On the contrary, $\mu_{d}<\mu_{n}+\mu_{p}$ below the crossing point at low densities but this is not allowed. All nucleons would form deuterons as neutron-proton bound states because the pure nucleonic system is less strongly bound than the condensate of deuterons. Considering more strongly bound bosonic clusters such as $\alpha$ particles, ${ }^{12} \mathrm{C}$, or ${ }^{16} \mathrm{O}$, the formation of these heavier clusters is expected. In fact, in the limit of infinite cluster mass, the density region of the liquid-gas phase transition is entered at low densities with the coexistence of macroscopic regions of low and high density nuclear matter.

In the practical application of an extended GRDF model with quasi-clusters at supra-saturation densities, a proper interpolation of the mass shift between the lowdensity limit from the microscopic Pauli blocking calculation and the high-density limit of the condensate model has to be introduced. As seen in Figure 4 it should be possible to find such a parametrization.

\section{Conclusions and outlook}

The GRDF model for nuclear matter can describe the dissolution of clusters with increasing density using the concept of cluster mass shifts. They are chosen customarily in such a way that the clusters, which have modified properties in the medium, do not appear at supra-saturation densities. The possibility of using these quasiclusters as an effective means to consider nuclear short-range correlation requires a change in the density dependence of the mass shifts. Exploratory calculations for quasi-deuterons in symmetric nuclear matter show that a much weaker increase of the mass shift is needed at high densities than in the present parametrization of the GRDF model. Since the case of zero temperature is studied, the deuteron fraction is determined by the density of the boson condensate that is incorporated into the model. At finite temperatures there will be a thermal contribution too because quasideuterons can be in excited states with a finite $\mathrm{cm}$ momentum. In contrast to the zero-temperature case, the single-particle momentum distribution of the nucleons will already develop a finite high-momentum tail because of the change in the distribution functions. The contribution of the quasi-deuterons will give only a certain fraction for high momenta partly arising from the neutron-proton short-range correlation inside the deuteron and partly from the thermal motion of the deuteron itself. 
For a future application of the cluster-correlation concept a suitable parametrization of the mass shifts has to be introduced that interpolates between the low-density limit constrained by microscopic many-body calculations and the high-density behavior derived in this work. Also a momentum dependence of the mass shift and a more involved dependence on the isospin asymmetry has to be considered. The precise form of the parametrization can be constrained by experimental results for the effective deuteron fraction in nuclear matter near saturation. The parametrization of the mass shift will only determine the mass fraction of the quasi-deuteron. To explore the single-particle momentum distribution in nuclear matter, explicit deuteron wave functions in the medium are needed. They can be obtained from microscopic calculations and will contain short-range correlations. Of course, the quasi-deuteron in the medium will look different than the deuteron in free space. Combining the relative nucleon-nucleon motion with the $\mathrm{cm}$ motion of the quasi-deuteron will give a contribution to the single-particle momentum distribution in nuclear matter. It is expected that a high-momentum tail emerges even at zero temperature. However, only more involved calculations can reveal how the momentum dependence will look like and how it compares to the experimentally observed $k^{-4}$ dependence. Work in this direction is in progress. In the present exploratory study only quasi-deuterons were considered. The effect of including heavier clusters and their relative importance can also be investigated, in particular the resulting differences in the high-momentum tail of the single-nucleon momentum distribution.

Open Access funding enabled and organized by Projekt DEAL. Support by the German Research Foundation and the Open Access Publishing Fund of Technical University of Darmstadt is acknowledged.

Open Access This is an open access article distributed under the terms of the Creative Commons Attribution License (https://creativecommons.org/licenses/by/4.0), which permits unrestricted use, distribution, and reproduction in any medium, provided the original work is properly cited.

Publisher's Note The EPJ Publishers remain neutral with regard to jurisdictional claims in published maps and institutional affiliations.

\section{References}

1. O. Hen, G. Miller, E. Piasetzky, L. Weinstein, Rev. Mod. Phys. 89, 045002 (2017)

2. E. Epelbaum, H. Hammer, U. Meissner, Rev. Mod. Phys. 81, 1773 (2009)

3. M. Oertel, M. Hempel, T. Klähn, S. Typel, Rev. Mod. Phys. 89, 015007 (2017)

4. P. Ring, P. Schuck, The Nuclear Many-Body Problem (Springer, Berlin, 1980)

5. A. Fetter, J. Walecka, Quantum Theory of Many-Particle Systems (McGraw-Hill, New York, 1971)

6. H. Hammer, S. König, U. van Kolck, Rev. Mod. Phys. 92, 025004 (2020)

7. M. Bender, P. Heenen, P. Reinhard, Rev. Mod. Phys. 75, 121 (2003)

8. M. Dutra, O. Lourenço, J. Sa Martins, A. Delfino, J. Stone, P. Stevenson, Phys. Rev. C 85, $035201(2012)$

9. R. Sellahewa, A. Rios, Phys. Rev. C 90, 054327 (2014)

10. M. Dutra, O. Lourenço, S. Avancini, B. Carlson, A. Delfino, D. Menezes, C. Providência, S. Typel, J. Stone, Phys. Rev. C 90, 055203 (2014)

11. S. Typel, G. Röpke, T. Klähn, D. Blaschke, H. Wolter, Phys. Rev. C 81, 015803 (2010)

12. E. Beth, G. Uhlenbeck, Physica 3, 729 (1936)

13. E. Beth, G. Uhlenbeck, Physica 4, 915 (1937) 
14. C. Horowitz, A. Schwenk, Nucl. Phys. A 776, 55 (2006)

15. E. O'Connor, D. Gazit, C. Horowitz, A. Schwenk, N. Barnea, Phys. Rev. C 75, 055803 (2007)

16. M. Schmidt, G. Röpke, H. Schulz, Ann. Phys. 202, 57 (1990)

17. G. Röpke, L. Münchow, H. Schulz, Nucl. Phys. A 379, 536 (1982)

18. G. Röpke, M. Schmidt, L. Münchow, H. Schulz, Nucl. Phys. A 399, 587 (1983)

19. G. Röpke, L. Münchow, H. Schulz, Phys. Lett. B 110, 21 (1982)

20. H. Stein, K. Morawetz, G. Röpke, Phys. Rev. A 55, 1945 (2000)

21. G. Röpke, N. Bastian, D. Blaschke, T. Klähn, S. Typel, H. Wolter, Nucl. Phys. A 897, $70(2013)$

22. G. Röpke, Phys. Rev. C 92, 054001 (2015)

23. M. Hempel, J. Schaffner-Bielich, Nucl. Phys. A 837, 210 (2010)

24. A. Raduta, F. Gulminelli, Phys. Rev. C 82, 065801 (2010)

25. M. Hempel, J. Schaffner-Bielich, S. Typel, G. Röpke, Phys. Rev. C 84, 055804 (2011)

26. V. Sagun, A. Ivanytskyi, K. Bugaev, I. Mishustin, Nucl. Phys. A 924, 24 (2014)

27. G. Röpke, Phys. Rev. C 79, 014002 (2009)

28. G. Röpke, Nucl. Phys. A 867, 66 (2011)

29. O. Hen et al., Science 346, 614 (2014)

30. H. Pais, S. Typel, in Nuclear particle correlations and cluster physics, edited by Wolf-Udo Schröder, (World Scientific, Singapore, 2017), pp. 95-133 